\title{
THE IMPACT OF $P$-HACKING ON "REDEFINE STATISTICAL SIGNIFICANCE"
}

\begin{abstract}
A recent proposal to "redefine statistical significance" (Benjamin et al. Nature
Human Behaviour, 2017) claims that lowering the default cutoff for statistical significance from 0.05 to 0.005 would "immediately improve the reproducibility of scientific research in many fields". Benjamin et al assert specifically that false positive rates would fall below $10 \%$ and replication rates would double under the lower cutoff. I analyze these claims here, showing how the failure to account for $P$-hacking and other widespread reporting issues leads to exaggerated and misleading conclusions about the potential impact of the Benjamin et al proposal. My analysis shows that the benefits of redefining statistical significance are far less certain than projected by Benjamin et al, with most plausible scenarios showing only a marginal improvement, and possible decline, in replication rate under the proposed 0.005 cutoff.
\end{abstract}

\section{INTRODUCTION}

The concept of replication reflects the basic scientific principle that a research finding 5 is reliable only if it can be verified by repeated, independent testing using accepted (and often the same or similar) experimental methods. The 'replication crisis' refers to the disproportionate amount of published scientific research that fails to live up to this principle, and the collective failure of leading scientists to resolve the problem. ${ }^{1}$ Without question, the issue is complicated, and it is serious, owing to a number of contributing factors that

10 lie outside the scope of ordinary scientific best practices, such as publication bias, lack of transparency and corruption in peer review, persistent abuse and misuse of statistical methods, and other so-called "questionable research practices" (QRPs) [Fraser, et al, 2018, John, et al, 2012]. Yet the problem persists, even with increased scrutiny and widespread efforts to remedy it through pre-registration [Chambers, 2014], bans on certain statistical

\footnotetext{
${ }^{1}$ The term 'reproducibility' is often treated as synonymous with 'replication', e.g., as in [Benjamin, et al, 2017], even though they have different meanings. Since I focus here on replication, I only use the term 'reproducibility' when quoting from sources that use the two words interchangeably. See http://www.replicability.tau.ac.il/index.php/replicability-in-science/replicability-vs-reproducibility.html for the distinction between these two terms.
} 
methods [Trafimow and Marks, 2015], and recommendations to 'redefine' [Benjamin, et al, 2017], 'remove' [Amrhein and Greenland, 2017], 'justify' [Lakens, et al, 2017], and 'abandon' [McShane, et al, 2017] the widely used benchmark of statistical significance.

I set out here to demonstrate how well-intentioned recommendations to improve replicability may not have their desired impact, and might even worsen the replication crisis. The main premise of my analysis is simple: a proposed solution to any problem (replication crisis or otherwise) cannot be assessed by a theory which tacitly assumes that the problem does not exist. In the specific case considered here, the problem is that the proportion of erroneous statistically significant P-values reported in the literature is much higher than standard statistical theory predicts. Therefore, when assessing the potential impact of any proposal to improve (i.e., decrease) the replication rate of findings obtained by significance testing, one cannot rely solely on the standard theory that is already known to be violated in light of the discrepancy between empirical and theoretical replication rates.

1.1. Proposal: Redefine statistical significance. To illustrate the above observations, I analyze the recent proposal to "redefine statistical significance" [Benjamin, et al, 2017] (henceforth abbreviated as 'RSS') by changing the default P-value cutoff for 'statistical significance' from 0.05 to 0.005 . I focus on the RSS proposal for expository reasons, as its assumed setting of hypothesis testing is widely used and is simple enough to convey the main message of this paper to a broad audience. I believe, however, that the spirit of my analysis - narrow attempts to address replication issues tend to understate the effects of $P$ hacking, publication bias, and other extra-scientific behaviors ${ }^{2}$ driven by built-in bias and adverse incentives, and that these behaviors are likely to persist, and may be exacerbated, by naive measures taken to counteract them-applies more broadly to many other proposals and in settings other than hypothesis testing. Since the discussion focuses on the RSS proposal, I have specialized many definitions, such as 'replication rate' in Section 2.2, to this setting. See [Mackey, 2012, Maxwell, et al, 2015] for a broader discussion of replication both in significance testing and beyond.

\footnotetext{
${ }^{2}$ Behaviors motivated by non-scientific considerations such as promotion, tenure, prestige, money, etc.
} 
As I lay out below, in failing to account for the impact of widespread departures from statistical protocol, including $P$-hacking as well as multiple testing, data snooping, cherry picking, publication bias, etc., on the output of statistical tests reported in the literature, the RSS authors arrive at an exaggerated and misleading assessment of the potential benefits of the lower significance cutoff. I explain the RSS authors' rationale for their claims, and argue that those claims are untenable in light of the many factors contributing to the replication crisis. At minimum, my analysis highlights important technical oversights in [Benjamin, et al, 2017] that call into question some of its more grandiose claims (e.g., that replicability will "immediately improve" and false positive rates will "fall by factors greater than two"). More broadly, the discussion here provides a cautionary tale when assessing new proposals to improve the practice of science, and in particular to address the replication crisis.

1.2. A note about $\boldsymbol{P}$-hacking. As $P$-hacking and other forms of statistical misuse are endemic to science at all levels, e.g., [Nuitjen, et al, 2016, Wicherts, et al, 2016], their role in the replication crisis cannot be overlooked when assessing proposals to resolve the issue. In ignoring these basic elements of the replication crisis, the argument in [Benjamin, et al, 2017] leads to overly optimistic projections about the "immediate" benefits of the 0.005 proposal. Because the specific kind of reporting problem is immaterial here, I streamline the discussion below and use the term ' $P$-hacking' as a catchall for these reporting issues, which include but are not limited to data snooping, data falsification, and outright fraud as well as "cherry-picking, hunting for significance, selective reporting, multiple testing and other biasing selection effects" [Mayo, 2017]. My generic use of the term P-hacking to refer to all of these behaviors is consistent with the terminology employed by other authors, e.g., [Simonsohn, et al, 2014, footnote 1, p. 534]. 1.3. Organization of article. I analyze the impact of the RSS proposal under three different scenarios. The first scenario, presented in Section 2, reproduces the argument in [Benjamin, et al, 2017], in which false positive rates between the 0.05 and 0.005 cutoff levels are compared under the standard theory of NHST. This first scenario does not account for the impact of $P$-hacking, loss of power, or other external factors on replication rate, even though it is well known that $P$-hacking (see Section 1.2) is a major contributing factor to the 


\begin{tabular}{|c||c|c|}
\hline & $H_{0}$ true & $H_{0}$ false \\
\hline Proportion & $\phi$ & $(1-\phi)$ \\
\hline \hline Reject & $\alpha \phi$ & $(1-\beta)(1-\phi)$ \\
\hline \multirow{2}{*}{ Not Reject } & $(1-\alpha) \phi$ & $\beta(1-\phi)$ \\
\hline
\end{tabular}

TABLE 1. Relative proportion of null hypotheses falling under each possible combination of true/false and reject/not reject for a family of statistical tests with Type-I error rate $\alpha$, Type-II error rate $\beta$, and prior odds $(1-\phi) / \phi$.

high rate of false positives in the literature, and relatedly the replication crisis. Therefore, when evaluating the effectiveness of any proposal to improve replicability, one must bear in mind that if the same incentives that currently motivate $P$-hacking (or related behaviors) remain after the proposal is implemented, then the same results (low replication rates) are likely to persist. I address this concern in the second scenario, presented in Section 3, in which I expand the analysis of Section 2 to account for $P$-hacking. In the presence of $P$ hacking, the analysis in Section 3 shows that the suggested benefits of the 0.005 proposal reported in Section 2 are severely diminished. The third scenario, presented in Section 4, expands the analysis in Section 3 by considering the possibility that the lower cutoff could lead to an increase in $P$-hacking (e.g., as researchers struggle to obtain publishable findings they will resort to $P$-hacking in order to overcome their difficulties) and an overall decrease in statistical power (as the increase in sample size needed to maintain the same level of power at the new significance level may not be attainable). Under these additional adverse conditions, the improvements to replication under the lower threshold are not only diminished (as reported in Section 3) but may be exacerbated, i.e., the replication rate could decrease under the lower threshold proposed in [Benjamin, et al, 2017]. In Section 5, I discuss the implications of these three scenarios in light of the specific claims made in [Benjamin, et al, 2017]. In Section 6, I make some concluding remarks. 


\section{Scenario 1: Classical Null Hypothesis Significance Testing (NHSt)}

Under the Null Hypothesis Significance Testing (NHST) paradigm [Neyman and Pearson, 1933], a null hypothesis $\left(H_{0}\right)$ is tested against an alternative hypothesis $\left(H_{1}\right)$ by computing a $P$-value, defined as the probability (under a true null hypothesis) that a certain test statistic attains a value as or more extreme than what is actually observed. Small $P$-values, which correspond to observations that are unlikely to have occurred if the null hypothesis were true, are interpreted as evidence against $H_{0}$. In fields adhering to the NHST paradigm, it is conventional to 'reject $H_{0}$ ' and confer the label of 'statistical significance' whenever a $P$-value falls below a pre-determined threshold $0<\alpha<1$. $^{3}$

Results obtained under the NHST protocol are prone to two types of statistical error:

- Type-I error: rejecting $H_{0}$ when $H_{0}$ is true; and

- Type-II error: failing to reject $H_{0}$ when $H_{0}$ is false.

Since when $H_{0}$ is true the corresponding $P$-value is distributed uniformly on $(0,1)$, the probability of a Type-I error (i.e., obtaining ' $P<\alpha$ ' when $H_{0}$ is true) is $\alpha$. For a given Type-I error probability, the Type-II error rate, denoted as $\beta$, is the probability of failing to reject a false null hypothesis. The power is the probability $1-\beta$ of correctly rejecting a false null hypothesis.

2.1. False positive rate. For a collection of statistical tests, the false positive rate (FPR) is the proportion of significant $P$-values (with $P<\alpha$ ) whose corresponding null hypothesis is true. So whereas the Type-I and Type-II error rates are the error probabilities for any given test conditioned on whether the null hypothesis is true or false, respectively, the FPR aggregates Type-I errors over all significant results in a collection of hypothesis tests. Thus, in addition to the Type-I and Type-II error rates of any given test, the FPR depends on the proportion $\phi$ of all tests in this collection for which the null hypothesis is true. In the Bayesian literature, the proportion $\phi$ is sometimes quoted in terms of the prior odds $(1-\phi) / \phi$ in favor of $H_{1}$, which intuitively conveys the odds that a 'randomly chosen' null

\footnotetext{
${ }^{3}$ Note that a $P$-value larger than $\alpha$ is not interpreted as evidence in favor of $H_{0}$. In the analysis below, I do not consider the so-called "accept-support" context, in which the researcher's hypothesis corresponds to $H_{0}$, instead of $H_{1}$, and large $P$-values are interpreted as support for retaining $H_{0}$.
} 

with Type-I and Type-II error probabilities $\alpha$ and $\beta$ and prior odds $(1-\phi) / \phi$, the false positive rate is given by

$$
\operatorname{FPR}(\alpha, \beta, \phi)=\frac{\alpha \phi}{\alpha \phi+(1-\beta)(1-\phi)}
$$

(Of the proportion $\phi$ of true null hypotheses, $\alpha$ are significant ('false positives'). Of the proportion $1-\phi$ of false null hypotheses, $1-\beta$ are significant ('true positives'). The false positive rate is the ratio of false positives $(\alpha \phi)$ to total positives (the sum of false positives and true positives given by $\alpha \phi+(1-\beta)(1-\phi)$ in the denominator $).)$

2.2. Replication Rate. A significant result $(P<\alpha)$ is replicable if it is verifiable by subsequent testing. Because attempts to replicate often appeal to the same or similar statistical methods as the initial study, they are prone to the same sources of statistical error. If, for example, a replication study has $90 \%$ power, then there is a $10 \%$ probability that an otherwise valid initial finding will fail to replicate in a single attempt. For simplicity, and to obtain a precise analysis of the replication rate for the purpose of illustration, I rule out the possibility of Type-I and Type-II error in replication attempts and assume that $100 \%$ of true positives and $0 \%$ of false positives are replicable. This assumption, which I call perfect replicability, treats replication as a property of the finding itself: if the finding is true, then it is replicable; if it is false, then it is not. Though an idealization-in practice, the replicability of any given finding depends on factors beyond whether the finding is true or false, such as power and effect size - perfect replicability aligns with the implicit assumptions made in [Benjamin, et al, 2017] and in analyses of the replication crisis more broadly. In principle, with enough resources and sufficient efforts dedicated to replication, it seems plausible to assume that false initial findings will be identified and true findings will be confirmed. Perfect replicability captures this idea by relating the replication rate directly to the false positive rate, as assumed in the analysis of [Benjamin, et al, 2017]. 
Plot of Power vs FPR (Figure 2 of RSS)

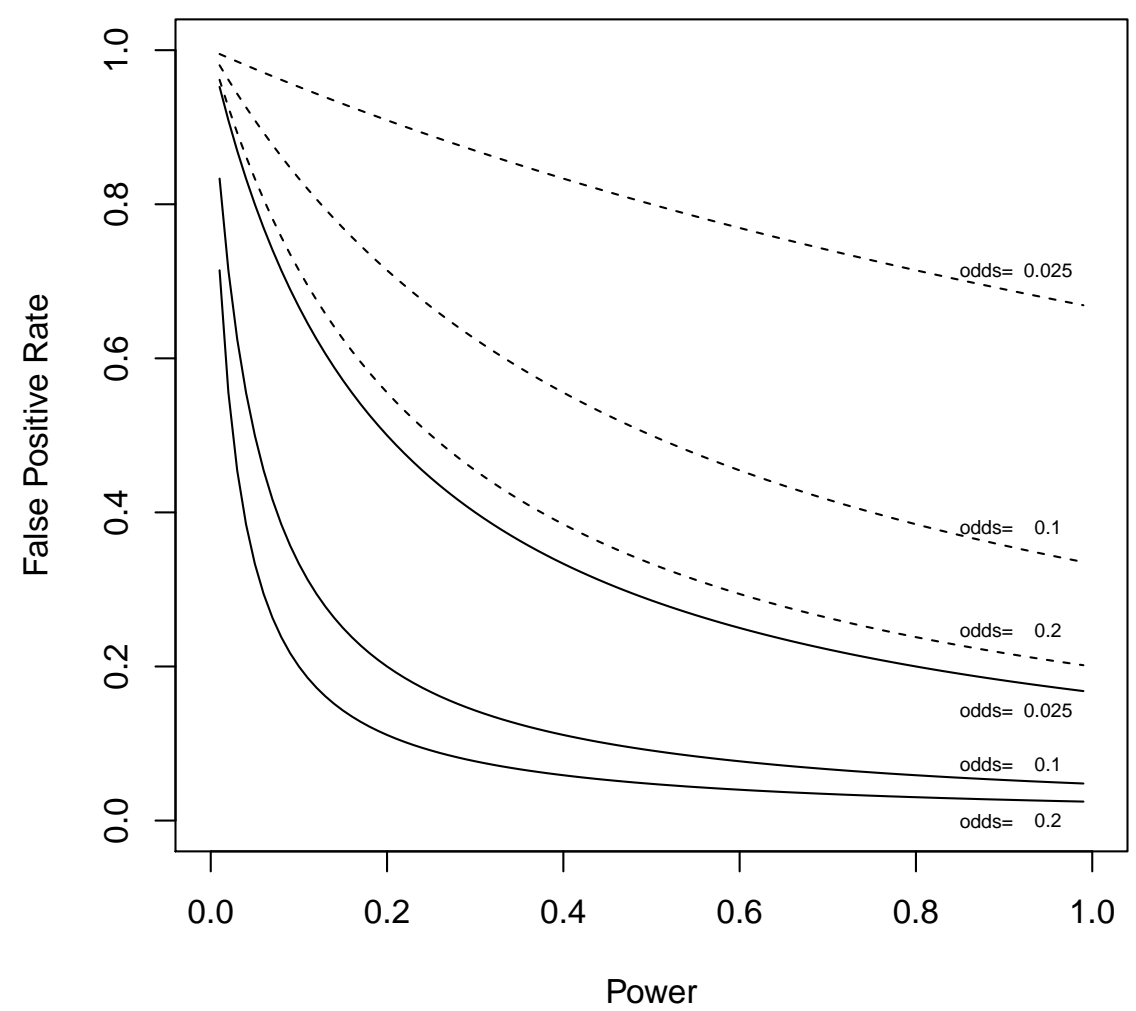

Figure 1. Plot of false positive rate (1) against power, as in [Benjamin, et al, 2017, Figure 2]. Dashed curves correspond to Type-I error rate 0.05. Solid curves correspond to Type-I error rate 0.005. Dashed and solid curves are labeled according to the assumed prior odds $(1-\phi) / \phi$ of $1 / 40,1 / 10$, and $1 / 5$.

Under perfect replicability, the replication rate for a family of tests with level $\alpha$, power 140

$$
\operatorname{RR}(\alpha, \beta, \phi)=\frac{(1-\beta)(1-\phi)}{\alpha \phi+(1-\beta)(1-\phi)} .
$$

Comparing (1) and (2), we observe the complementary relationship between replication rate and false positive rate,

$$
\operatorname{RR}(\alpha, \beta, \phi)=1-\operatorname{FPR}(\alpha, \beta, \phi) .
$$


2.3. Reproducing the RSS analysis. The analysis presented in support of the RSS proposal [Benjamin, et al, 2017] relies on the calculations of FPR and RR in (1) and (2) above. I reproduce the RSS analysis here for completeness.

The RSS analysis cites the relative difference in false positive rate between significance levels 0.05 and 0.005 for a fixed prior odds and level of power in support of its proposal to redefine statistical significance. The analysis first observes that FPR exceeds $33 \%$ for significance level 0.05 and prior odds 1/10 "regardless of the level of statistical power". (This observation corresponds to the middle dashed line in Figure 1, labeled 'odds $=0.1$ ', which lies above 0.33 for all levels of power.) The authors continue, "Reducing the threshold to 0.005 would reduce this minimum false positive rate to $5 \%$. Similar reductions in false positive rates would occur over a wide range of statistical powers". These claims are based on comparisons between the dashed and solid lines labeled 'odds $=0.1$ ' in Figure 1.

The RSS analysis goes on to cite empirical evidence in support of its more specific claims that decreasing the significance threshold would "immediately improve" reproducibility. The authors specifically refer to two recent replication projects, one in psychology [Open Science Collaboration, 2015] and another in experimental economics [Camerer, et al, 2016], in which the replication rate for initial studies with $P<0.005$ was "roughly double" those with $P$-value in the intermediate range $0.005<P<0.05$.

I analyze the argument and conclusions from [Benjamin, et al, 2017] further in Section 5. But first, to understand the RSS proposal in the proper context, it is important to consider the scope of the RSS argument, and the extent to which the theoretical values of FPR and the empirical comparison of replication rates for results significant at levels 0.05 and 0.005 (as reported in [Camerer, et al, 2016, Open Science Collaboration, 2015]) are salient for assessing the effectiveness of the proposed change in significance level. Crucial to this assessment is the fact that both the theoretical calculations in Figure 1 and the RSS interpretation of the empirical observations cited in [Benjamin, et al, 2017] rely on assumptions that are clearly violated in light of the replication crisis. In particular, the RSS analysis depends on the theoretical calculations of FPR in (1), which fail to account for the practical issues, referred to generically here as $P$-hacking (see Section 1.2), that are 
central to the replication crisis. In citing the theoretical calculations in Figure 1 to support its proposal, the RSS authors thus appeal to the same theory which is known to be invalid by the mere existence of the replication crisis they are seeking to improve. To accurately assess the impact of the lower significance cutoff on the replication crisis, the calculations of FPR and RR in (1) and (2) must be refined to account for the fact that the classical theory of NHST is known to fail in this setting.

\section{Scenario 2: NHST with $P$-hacking}

When analyzing the impact of $P$-hacking on replication, it is important to distinguish understate the impact of $P$-hacking on false positive and replication rates.

To account for the impact of $P$-hacking on the replication rate, I expand the framework of Section 2 by distinguishing between sound and unsound P-values: 
- A sound $\boldsymbol{P}$-value is one for which the standard interpretation is valid (i.e., the probability, under the null hypothesis, that the test statistic attains a value as extreme or more extreme than observed).

- An unsound (or hacked) $\boldsymbol{P}$-value is any $P$-value that does not warrant the above interpretation.

For us, a $P$-value is sound whenever it is obtained by proper application of NHST protocol. Sound $P$-values are not tainted by selective reporting, data snooping, cherry picking, or any of the other issues contributing to the higher than anticipated rate of false positives in the published literature. For sound $P$-values, Type-I error is the only source of false positives, and the theory of Section 2 applies. Unsound $P$-values, on the other hand, are affected by the above mentioned reporting issues, and thus contribute to the overall higher proportion of false positives than the classical theory can explain. By accounting for both sound and unsound $P$-values in the analysis of FPR, I find that even a relatively small proportion of unsound $P$-values can have a substantial impact on the projected replication rate.

3.1. False positive rate and replication rate under $\boldsymbol{P}$-hacking. The classification of $P$-values as sound and unsound identifies two different regimes for computing FPR. We write $0<h<1$ to denote the $P$-hacking rate, i.e., the proportion of unsound $P$-values (those obtained in violation of standard NHST protocol) among all $P$-values under consideration. With $0<\alpha<1$ fixed as a baseline, we assume that the entire proportion $h$ is significant at level $\alpha$ when the prevailing significance cutoff is $\alpha$. The remaining $P$-values (proportion $220 \quad 1-h$ of all $P$-values) are sound and behave according to the analysis in Section $2 .{ }^{4}$ With $P$-hacking (and other reporting defects) accounted for through $h$, the false positive rate is computed by

$$
\operatorname{FPR}(\alpha, \beta, \phi ; h)=\frac{\alpha(1-h) \phi+h}{\alpha(1-h) \phi+(1-\beta)(1-h)(1-\phi)+h} .
$$

\footnotetext{
${ }^{4}$ I disregard attempts at $P$-hacking which fail to achieve $P<\alpha$. Since these $P$-values are never labeled 'significant', they do not appear in the scientific literature and therefore do not contribute to the replication crisis. As mentioned before (see footnote 3), I also do not consider the "accept-support" context of significance testing.
} 
(Contributing to the numerator are (i) the proportion $\alpha(1-h) \phi$ of false positives under the classical (i.e., sound) NHST paradigm from Section 2 and (ii) the proportion $h$ of significant $P$-values obtained by $P$-hacking.) Under the assumption of perfect replicability, we again observe the complementary relationship between false positive rate and replication rate:

$$
\operatorname{RR}(\alpha, \beta, \phi ; h)=1-\operatorname{FPR}(\alpha, \beta, \phi ; h)=\frac{(1-\beta)(1-h)(1-\phi)}{\alpha(1-h) \phi+(1-\beta)(1-h)(1-\phi)+h} .
$$

Comparing (4) to the calculation of FPR used in the RSS analysis (equation (1) in Section 2.3) illustrates precisely how the RSS analysis ignores the effects of $P$-hacking on replication rate: by setting $h=0$ in (4) we recover (1). Table 2 summarizes the factors contributing to the calculations in (4) and (5).

I point out here some similarities between the calculations in (4) and (5), which compute false positive rate and replication rate in the presence of $P$-hacking, and those for positive predicted value (PPV) in [Ioannidis, 2005], which compute the probability that a reported research finding is true in the presence of reporting bias. The latter calculations were put forward in support of Ioannidis's assertion that "most published research findings are false". Whereas [Ioannidis, 2005] focuses on the validity of reported findings, the emphasis here is on replication, and furthermore on changes to the replication rate upon decreasing the significance level. As Ioannidis's argument in [Ioannidis, 2005] is often cited as a seminal work on the replication crisis, it is noteworthy that the RSS analysis ignores Ioannidis's considerations of bias in their assessment of the 0.005 cutoff on replication rates. ${ }^{5}$

Additional considerations of $P$-hacking beyond the dichotomous decision of whether or not to reject $H_{0}$ figure into the replication problem. Since researchers do not know whether they are testing a genuine effect, there are also hacked $P$-values which correspond to true effects. From the perspective of FPR alone, $P$-hacking in such instances is beneficial, in effect serving as an artificial 'power booster' for false null hypotheses that would otherwise go undetected. In such cases, the $P$-value is technically unsound and the effect size inflated, even though subsequent attempts to replicate will be successful under the assumption of perfect

\footnotetext{
${ }^{5}$ This is especially noteworthy given that Ioannidis is one of the many co-authors of [Benjamin, et al, 2017].
} 


\begin{tabular}{|c||c|c|c|}
\hline \multicolumn{1}{|c||}{} & \multicolumn{2}{|c|}{$H_{0}$ true } & $H_{0}$ false \\
\hline Proportion & $\begin{array}{c}\text { Sound } \\
\phi(1-h)\end{array}$ & $\begin{array}{c}\text { Unsound } \\
h\end{array}$ & $\begin{array}{c}\text { Sound } \\
(1-\phi)(1-h)\end{array}$ \\
\hline \hline Reject & $\alpha(1-h) \phi$ & $h$ & $(1-\beta)(1-h)(1-\phi)$ \\
\hline Not Reject & $(1-\alpha)(1-h) \phi$ & 0 & $\beta(1-h)(1-\phi)$ \\
\hline
\end{tabular}

TABLE 2. Relative proportions of null hypotheses corresponding to true/false positives/negatives in the presence of $P$-hacking. The proportion $1-h$ of sound tests has Type-I error rate $\alpha$, Type-II error rate $\beta$, and prior odds $(1-\phi) / \phi$. Setting $h=0$ recovers the proportions in Table 1 .

replicability. Such considerations have a complex bearing on replication issues that extend beyond the simplified setting of the RSS proposal under perfect replicability discussed here.

3.2. $P$-value distributions. In addition to ignoring $P$-hacking in its theoretical analysis, RSS cites empirical evidence about the relative replication rates of $P$-values in the ranges $P<0.005$ and $0.005<P<0.05$. In presenting this data as evidence in support of its proposal, however, the RSS authors once again fail to account for the distinction between policy and protocol. Since the empirical replication rates cited in [Benjamin, et al, 2017] include both sound and unsound $P$-values, the impact of the 0.005 significance level cannot be properly assessed without accounting for the dependence of the magnitude of unsound $P$-values on the prevailing significance threshold. Empirical studies, e.g., [Head, et al, 2015, Mariscampo and Lalande, 2012, Simonsohn, et al, 2014], find that unsound P-values tend to cluster disproportionately close to the significance cutoff (i.e., tend to lie just below 0.05). Though some authors [Bishop and Thompson, 2016, Hartgerink, 2017, Hartgerink, et al, 2016] have raised concerns about these claims, these criticisms do not seem to have a direct bearing on our analysis, which primarily depends on the $P$-value distributions in the regions $P<0.005$ and $0.005<P<0.05$, neither of which is confined to $P$-values falling just below 0.05 . 
The fact that hacked $P$-values tend to lie in the region $0.005<P<0.05$ when the significance cutoff is 0.05 offers partial explanation for why the replication rate of $P$-values with $P<0.005$ is much higher than those with $0.005<P<0.05$ when the prevailing significance cutoff is 0.05 . But when analyzing the impact of the lower significance policy in the presence of $P$-hacking, we must note that while the behavior of sound $P$-values can be treated as absolute - by virtue of being sound their behavior is unaffected by policy changes - the behavior of unsound $P$-values must be interpreted relative to the prevailing policy. This dependence on policy might explain why unsound $P$-values tend to lie just below 0.05 when the cutoff is 0.05 , and suggests that many of these same unsound $P$-values would tend to lie just below 0.005 when the cutoff changes to 0.005 , as a result of more aggressive $P$-hacking.

We also cannot ignore that the scientific teams behind successfully hacked $P$-values have been successful not only in obtaining a significant $P$-value at the prevailing significance threshold but also in justifying why their hacked $P$-value corresponds to a finding worthy of publication. When the significance threshold changes, we should expect that these same scientists will continue to be successful in finding ways to obtain significant, publishable results. Thus, when assessing the impact of a regime change on false positives and reproducibility, we must take into account that some, and perhaps many, of the hacked $P$-values that currently lie in the region $0.005<P<0.05$ will persist (and continue to be significant at the lower $P<0.005$ level) after the policy for conferring statistical significance is changed to 0.005. (Before moving on, I comment briefly about the RSS authors' insistence that their proposal is not intended as a threshold for publication. Even if the 0.005 cutoff is not adopted as a publication policy, the allure of "statistical significance" is already deeply ingrained in the mindset of many scientists. It is naive to expect scientists to stop pursuing "significant" findings and to instead settle for being allowed to publish results that are merely "suggestive", as the RSS authors recommend for $P$-values in the range $0.005<P<0.05$. And, because scientists will keep pursuing significance, journal editors will eventually come around to adopting 0.005 as the de facto publication standard. The cycle will continue for as long as the dichotomous labels of significance and non-significance 
Under $\alpha / c$ cutoff

\begin{tabular}{|c||c|c|c|}
\hline \multicolumn{1}{|c||}{} & \multicolumn{2}{|c|}{$H_{0}$ true } & $H_{0}$ false \\
\hline Proportion & $\begin{array}{c}\text { Sound } \\
\phi(1-h)\end{array}$ & $\begin{array}{c}\text { Unsound } \\
h\end{array}$ & $\begin{array}{c}\text { Sound } \\
(1-\phi)(1-h)\end{array}$ \\
\hline \hline Reject & $(\alpha / c)(1-h) \phi$ & $h \psi_{\alpha / c}(\alpha / c)$ & $(1-\beta)(1-h)(1-\phi)$ \\
\hline Not Reject & $(1-\alpha / c)(1-h) \phi$ & $h\left(1-\psi_{\alpha / c}(\alpha / c)\right)$ & $\beta(1-h)(1-\phi)$ \\
\hline
\end{tabular}

TABLE 3. Relative proportions of null hypotheses corresponding to true/false positives/negatives in the presence of $P$-hacking with persistence $\psi_{\alpha / c}(\alpha / c)$ at level $\alpha / c$. The proportion $1-h$ of sound tests has Type-I error rate $\alpha / c$, Type-II error rate $\beta$, and prior odds $(1-\phi) / \phi$. From this, we recover Table 2 by setting $c=1$ and $\psi_{\alpha}(\alpha)=1$, and we recover Table 1 by setting $h=0$.

remain in the lexicon, regardless of whether or not these labels are used as an explicit publication criterion. The RSS proposal does nothing to counteract this trend, and in fact furthers the trend by suggesting a new criterion for assigning the dichotomous labeling.)

To account for the persistence of $P$-hacking under the lower cutoff, we write $\psi_{\alpha / c}(x), 0<$ $x<1$, to denote the proportion of hacked $P$-values in the range $(0, x)$ when the significance cutoff is $\alpha / c$, for $c \geq 1$. For $c \geq 1, \psi_{\alpha / c}(\alpha / c)$ is the proportion of all hacked $P$-values that continue to be significant (i.e., 'persist') when the threshold is decreased to $\alpha / c$. Since the behavior of hacked $P$-values depends on the cutoff, we cannot naively estimate $\psi_{\alpha / c}(\alpha / c)$ by $\psi_{\alpha}(\alpha / c)$ (i.e., the proportion of hacked $P$-values that lie below $\alpha / c$ when the significance cutoff is $\alpha$ ), as the RSS analysis does in arriving at the claimed 2-to-1 improvement in replication rate under the 0.005 cutoff. Instead, when analyzing the potential impact of the lower cutoff, we consider all possible values in the range $\psi_{\alpha}(\alpha / c) \leq \psi_{\alpha / c}(\alpha / c) \leq \psi_{\alpha}(\alpha)$ by interpolation,

$$
\psi_{\alpha / c}(\alpha / c)=\pi \psi_{\alpha}(\alpha)+(1-\pi) \psi_{\alpha}(\alpha / c)
$$


for $0<\pi<1$. We call $\pi$ the persistence parameter and sometimes refer to $\psi_{\alpha / c}(\alpha / c)$ simply as the persistence at level $\alpha / c$, as both quantify the extent to which hacked $P$-values 'persist' after a change in cutoff from $\alpha$ to $\alpha / c$.

In fixing $\alpha$ as the baseline, we set $\psi_{\alpha}(\alpha)=1$ so that the proportion of unsound significant $P$-values at level $\alpha$ equals $h \psi_{\alpha}(\alpha)=h$ as in Table 2. With $h$ assumed to be independent of the cutoff, the proportion of all hacked $P$-values that are significant at the new $\alpha / c$ level is given by $\psi_{\alpha / c}(\alpha / c)$, as reflected in the updated proportions of Table 3 . The cutoff-dependent distribution $\psi$ of unsound $P$-values features in the calculation of FPR and RR by

$$
\begin{aligned}
\operatorname{FPR}(\alpha / c, \beta, \phi ; h, \psi) & =\frac{(\alpha / c) \phi(1-h)+h \psi_{\alpha / c}(\alpha / c)}{(\alpha / c) \phi(1-h)+h \psi_{\alpha / c}(\alpha / c)+(1-\beta)(1-\phi)(1-h)} \quad \text { and } \\
\operatorname{RR}(\alpha / c, \beta, \phi ; h, \psi) & =\frac{(1-\beta)(1-\phi)(1-h)}{(\alpha / c) \phi(1-h)+h \psi_{\alpha / c}(\alpha / c)+(1-\beta)(1-\phi)(1-h)} .
\end{aligned}
$$

310 Note the relationship between the calculations of false positive and replication rates in (4) and (5) and those in (7) and (8). Importantly, the calculations in (4)-(5) are the rates under the current significance cutoff $\alpha$ with a $P$-hacking rate of $h$, while those in (7)-(8) are the rates under the new significance cutoff $\alpha / c$ with a proportion $\psi_{\alpha / c}(\alpha / c)$ of hacked $P$-values assumed to persist at the lower significance level. Setting $c=1$ and $\psi_{\alpha / c}(\alpha / c)=1$

315 in (7)-(8) recovers (4)-(5), respectively.

3.3. Analysis with $\boldsymbol{P}$-hacking. Figures 2 and 3 plot the false positive rate (FPR) versus statistical power for different combinations of significance level and $P$-hacking rate $h$, under assumed prior odds of $1 / 10,1 / 5$, and $1 / 40$, respectively. The solid lines, which correspond to the traditional FPR in the absence of $P$-hacking (see equation (1)), suggest a substantial improvement to FPR under the reduced significance level: at $80 \%$ power, FPR is projected to decrease from 0.38 to 0.06 with prior odds $1 / 10$; and at $50 \%$ power, FPR is projected to decrease from 0.50 to 0.09. (These numbers can be read from the solid lines in Figure 2, or by referring to the original RSS plot reproduced in Figure 1.) But if $15 \%$ of all $P$-values are hacked, ${ }^{6}$ then FPR would decrease from 0.75 to 0.71 (at $80 \%$ power) and from 0.83 to 0.80

\footnotetext{
${ }^{6}$ In Section 5, we estimate that between $3 \%$ and $15 \%$ of all $P$-values are hacked based on data from a replication study in psychology [Open Science Collaboration, 2015]. These estimates are used here for illustration and should not be interpreted as a definitive range for the actual rate of $P$-hacking in the published literature.
} 


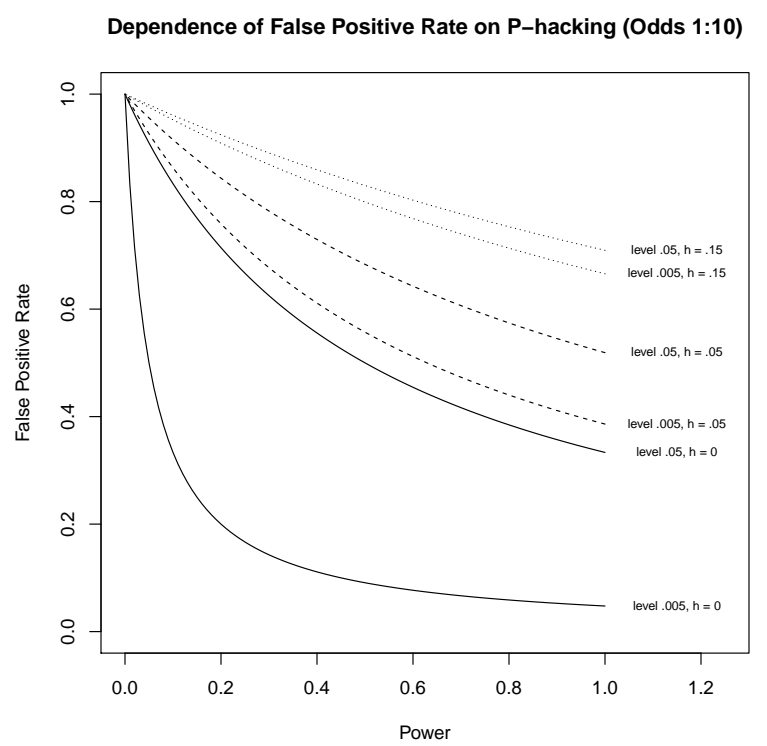

Figure 2. False positive rate (4) for different significance levels ( $\alpha=$ $0.05,0.005)$ and $P$-hacking rates $(h=0,0.05,0.15)$ with $1 / 10$ prior odds. Solid lines correspond to false positive rate without $P$-hacking; dashed lines to FPR with $h=0.05$ (i.e., $5 \%$ of all $P$-values are hacked); and dotted lines to FPR with $h=0.15$.

(at $50 \%$ power), just a $4-5 \%$ improvement. And even if only $3 \%$ of $P$-values are hacked, the FPR would only improve from 0.51 to 0.33 (at $80 \%$ power) and from 0.63 to 0.44 (at $50 \%$ power). But regardless of how much the FPR improves in relative terms, the end result (33\%-80\% false positives) is bound to remain much higher than the $6-9 \%$ level suggested by the analysis in [Benjamin, et al, 2017]. Figure 3 illustrates the same effect when the prior odds are assumed to be $1 / 5$ or $1 / 40$. In general, the suggested benefits of lowering the cutoff from 0.05 to 0.005 are mitigated substantially (for a given level of power and a given prior odds) in the presence of $P$-hacking.

The bottom solid line (labeled ' $h$ ' $=0$ ') in each of the panels of Figure 4 shows how the FPR varies as a function of persistence when the $P$-hacking rate $h$ is assumed to be 0.05 or 0.15. (The meaning of $h^{\prime}$ is explained in the more general setup of the next section, but plays no role in the present analysis.) These figures assume that both the overall prevalence of $P$-hacking, as measured by $h$, and the level of power, as measured by $1-\beta$, remain constant upon changing the cutoff from 0.05 to 0.005. As we see by comparing the top 

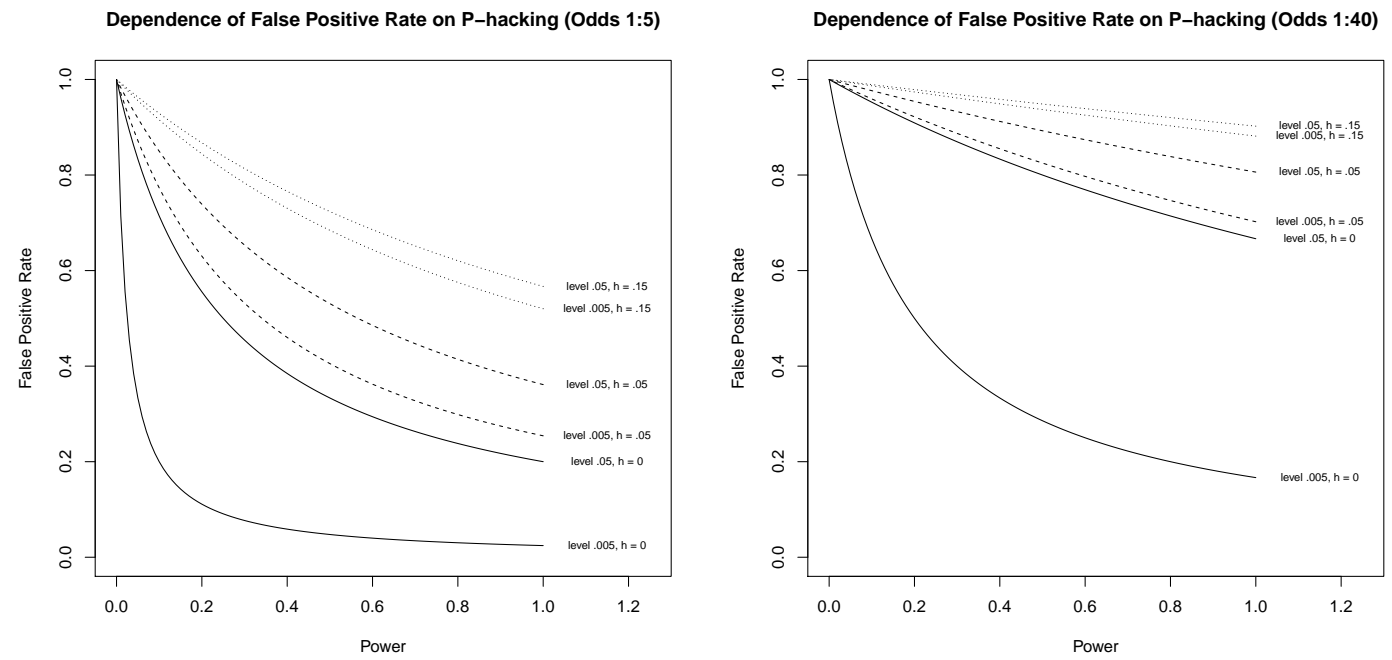

Figure 3. False positive rate (4) for different significance levels $(\alpha=$ $0.05,0.005)$ and $P$-hacking rates $(h=0,0.05,0.15)$ with assumed prior odds of $1 / 5$ (left) and $1 / 40$ (right). Solid lines correspond to false positive rate without $P$-hacking; dashed lines to FPR with $h=0.05$ (i.e., $5 \%$ of all $P$ values are hacked); and dotted lines to FPR with $h=0.15$.

and bottom rows of Figure 4, the results are qualitatively similar across different levels of power in that the FPR is substantially higher than the ideal theoretical value (the bottom dotted line with $h=0$ ) as long as persistence is non-negligible (larger than 0.20 or so when $h=0.05$ and larger than 0.10 when $h=0.15$ ). And if persistence is relatively high (larger than 50\%), then any perceived improvements to FPR under the lower threshold are greatly reduced. I discuss more specific implications for the RSS proposal in Section 5.

\section{Scenario 3: NHST with adverse P-hacking}

The persistence parameter in the FPR calculation of (7) accounts for the degree to which unsound $P$-values at the $\alpha$ significance level will continue to be significant (perhaps because of further $P$-hacking) when the cutoff policy for conferring statistical significance is changed to $\alpha / c$. The analysis in Section 3, therefore, assumes no new sources of $P$-hacking at the lower significance level - any $P$-values that were sound under the $\alpha$ policy remain sound under the new $\alpha / c$ policy - and that the same level of power can be maintained at the lower cutoff value. Some authors, e.g., [Amrhein and Greenland, 2017, McShane, et al, 2017], have 
raised the concern that the more stringent significance policy could increase the amount of $P$-hacking, as scientists will face the same pressures to publish despite greater difficulty to obtain significant findings, which would lead to an overall decrease in statistical power as many scientists lack the funding and other resources needed to increase sample sizes so that the same level of power can be maintained. A decrease in power would be consistent with well documented sub-optimal practices of NHST, as the emphasis on significance makes running smaller, underpowered studies a more effective strategy than larger, high-powered studies [Bakker, et al, 2012]. Decreasing the significance cutoff to 0.005 can be expected to further incentivize this sub-optimal practice. With lower power, the number of TypeII errors will go up, which will in turn increase the need to $P$-hack genuine effects. As mentioned above, this additional $P$-hacking serves as an artificial 'power booster' for tests that can no longer achieve a sufficient level of power to obtain statistical significance. But even if the direction of the effect is genuine, this leads to bias and inflation in estimated effect sizes which have a complex bearing on false positive rates and replicability. In this section, we analyze the potential impact of these possibilities by considering how the lower significance threshold could further contribute to the replication crisis by leading to lower powered studies (higher type-II error) and increased $P$-hacking (higher $h$ ).

In addition to the persistence parameter $\psi_{\alpha / c}(\alpha / c)$, which measures the proportion of unsound, significant $P$-values at level $\alpha$ that remain significant under the lower $\alpha / c$ cutoff, there is the potential increase in overall $P$-hacking attempts from $h$ to $h+h^{\prime}$, with $0 \leq h^{\prime} \leq$ $1-h$ measuring the additional proportion of unsound $P$-values caused by the change in cutoff to $\alpha / c$. We assume for the sake of illustration that the entire proportion $h^{\prime}$ of these new unsound $P$-values is significant at the $\alpha / c$ level after the change in cutoff policy. ${ }^{7}$ With the additional factor of $h^{\prime}$, we compute false positive rate and replication rate (again under

\footnotetext{
${ }^{7}$ As noted when $h$ was originally introduced in Section 3 , we disregard new $P$-hacking attempts at the $\alpha / c$ level that fail to produce a significant result, because these failed attempts do not contribute to the replication crisis.
} 
Under $\alpha / c$ cutoff

\begin{tabular}{|c||c|c|c|}
\hline \multicolumn{1}{|c||}{} & \multicolumn{2}{|c|}{$H_{0}$ true } & $H_{0}$ false \\
\hline Proportion & $\begin{array}{c}\text { Sound } \\
\phi\left(1-h-h^{\prime}\right)\end{array}$ & $\begin{array}{c}\text { Unsound } \\
h+h^{\prime}\end{array}$ & $\begin{array}{c}\text { Sound } \\
(1-\phi)\left(1-h-h^{\prime}\right)\end{array}$ \\
\hline \hline Reject & $(\alpha / c)\left(1-h-h^{\prime}\right) \phi$ & $h \psi_{\alpha / c}(\alpha / c)+h^{\prime}$ & $(1-\beta)\left(1-h-h^{\prime}\right)(1-\phi)$ \\
\hline Not Reject & $(1-\alpha / c)\left(1-h-h^{\prime}\right) \phi$ & $h\left(1-\psi_{\alpha / c}(\alpha / c)\right)$ & $\beta\left(1-h-h^{\prime}\right)(1-\phi)$ \\
\hline
\end{tabular}

TABLE 4. Relative proportions of null hypotheses corresponding to true/false positives/negatives in the presence of $P$-hacking with persistence $\psi_{\alpha / c}(\alpha / c)$ at level $\alpha / c$ and proportion $0 \leq h^{\prime} \leq 1-h$ of new unsound $P$ values under the $\alpha / c$ cutoff. The proportion $1-h-h^{\prime}$ of sound tests has Type-I error rate $\alpha / c$, Type-II error rate $\beta$, and prior odds $(1-\phi) / \phi$. From this, we recover Table 3 by setting $h^{\prime}=0$.

the assumption of perfect replicability) by

$$
\begin{aligned}
& \operatorname{FPR}\left(\alpha / c, \beta, \phi ; h, h^{\prime}, \psi\right)= \\
& \quad=\frac{(\alpha / c) \phi\left(1-h-h^{\prime}\right)+h \psi_{\alpha / c}(\alpha / c)+h^{\prime}}{(\alpha / c) \phi\left(1-h-h^{\prime}\right)+h \psi_{\alpha / c}(\alpha / c)+(1-\beta)(1-\phi)\left(1-h-h^{\prime}\right)+h^{\prime}}
\end{aligned}
$$

and

$$
\begin{aligned}
& \operatorname{RR}\left(\alpha / c, \beta, \phi ; h, h^{\prime}, \psi\right)= \\
& \quad=\frac{(1-\beta)(1-\phi)\left(1-h-h^{\prime}\right)}{(\alpha / c) \phi\left(1-h-h^{\prime}\right)+h \psi_{\alpha / c}(\alpha / c)+(1-\beta)(1-\phi)\left(1-h-h^{\prime}\right)+h^{\prime}} .
\end{aligned}
$$

The components of these calculations are summarized in Table 4.

The red solid lines in Figure 4 show how the analysis in Section 3 is affected by additional $P$-hacking through $h^{\prime}$, and that the relative impact of $h^{\prime}$ on FPR is consistent across different levels of power (assuming that power is held fixed under both new and old significance levels). Notice in particular that when $h=0.05$, if the lower cutoff causes the prevalence of $P$-hacking to double (i.e., $h^{\prime}=0.05$ ), then the FPR will remain roughly the same both 

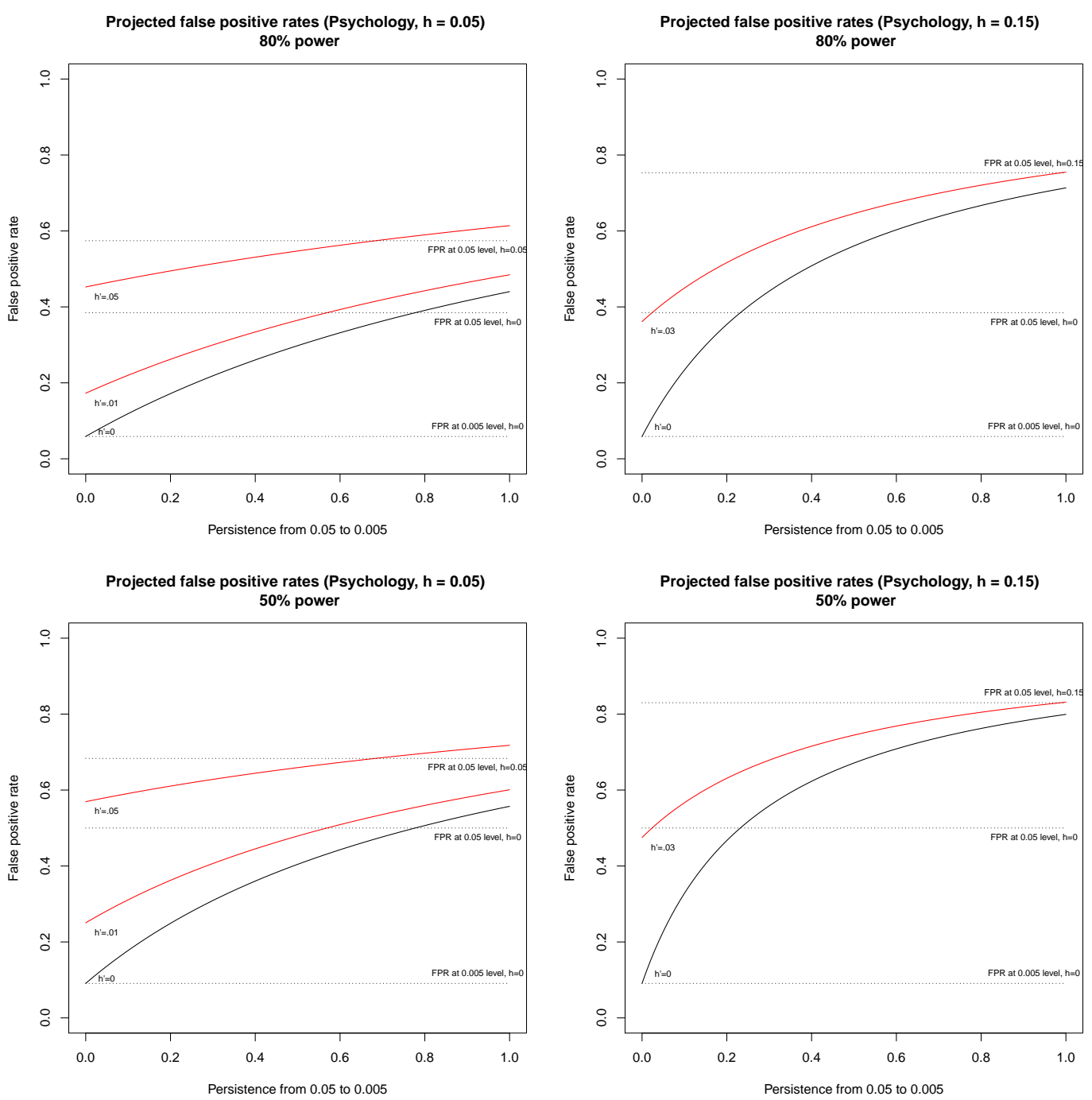

FiguRE 4. Solid lines show the projected FPR at level 0.005 for $h=0.05$ (left) and $h=0.15$ (right) over the full range $0<\pi<1$ of persistence with different values of $h^{\prime}$. All plots assume prior odds $1 / 10$, with top row assuming $80 \%$ power and bottom row assuming 50\% power. Top dotted line in each plot is FPR computed at extreme values of the estimated range ( $h=0.05$ on left, $h=0.15$ on right). In both plots, the middle dotted line is FPR at level 0.05 and $h=0$ and the bottom dotted line is FPR at level 0.005 and $h=0$. Red solid lines correspond to situations for which $h^{\prime}$ is non-zero, as in Table 4.

before and after the change in cutoff, regardless of the level of persistence. And if persistence is moderate to high, then the FPR could increase under the 0.005 significance policy. But even the calculations in Figure 4 are optimistic, as they assume that power remains 

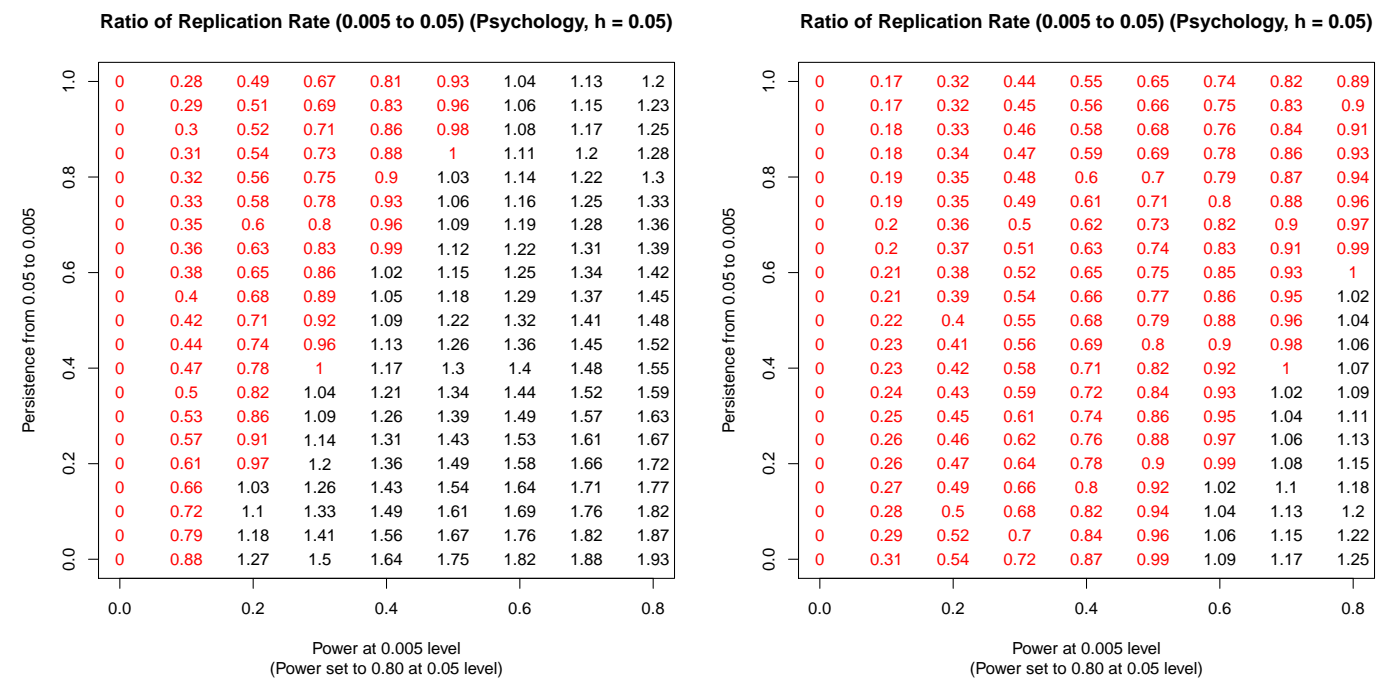

FiguRE 5. Ratio of RR under 0.005 and 0.05 cutoffs based on the psychology replication data for different combinations of power $1-\beta$ and persistence $\psi_{0.005}(0.005)$ :

$$
\frac{\operatorname{RR}\left(0.005, \beta, 10 / 11 ; h, h^{\prime}, \psi_{0.005}(0.005)\right)}{\operatorname{RR}(0.05,0.2,10 / 11 ; h, 0,1)} .
$$

Power is set to $80 \%$ at the 0.05 level, but is assumed to vary over the entire range $0 \leq \beta \leq 1$ at the 0.005 level. The $P$-hacking rate is set to $h=0.05$ on left and right, while the additional $P$-hacking $h^{\prime}$ introduced at the new level is set to 0.01 on the left and 0.05 on the right. Ratios less than 1 are colored red, indicating that replication rate is worse under the 0.005 cutoff.

constant after switching from 0.05 to 0.005 . In practice, it is unlikely that whatever level of power is currently achievable at the 0.05 level (whether $80 \%, 50 \%$, or something else) can be maintained upon switching to the more stringent 0.005 level. To achieve the same power at the lower significance level, the sample size will need to increase, in some cases prohibitively due to limited funding or a limited pool of available subjects, as noted, e.g., in [Hamlin, 2017]. Figure 5 shows the sensitivity of replication rate to adverse $P$-hacking $h^{\prime}$ and decreased power under the new threshold. I discuss specific implications for the RSS proposal in the next section.

\section{Assessing the impact of "Redefining Statistical Significance"}

In view of Sections 2-4, I now address specific claims made in [Benjamin, et al, 2017]. For simplicity, I evaluate these claims under the assumed power of $0.80(\beta=0.20)$ and 0.50 
$(\beta=0.50)$ at significance level 0.05 . (The choice of $80 \%$ power reflects the conventional "gold

\footnotetext{
${ }^{8}$ The prior odds can be expected to decrease for the same reasons cited in Section 4 to explain why $P$-hacking will increase and power will decrease under the lower significance cutoff. Since, under the lower cutoff, it will be more challenging to obtain significant findings, scientists may respond by testing many more spurious hypotheses in search of significant results. Such a decrease in prior odds would lead to an increase in false positive rate even in the absence of any other types of $P$-hacking. I do not consider this possibility any further here.
} 
other discipline. The estimated range $0.05<h<0.15$ serves merely as a guideline in the upcoming analysis of the main claims put forward in [Benjamin, et al, 2017].

(i) The observation (partially reconstructed in Figure 2) that FPR is unacceptably high (larger than $33 \%$ at all levels of power) under the 0.05 cutoff, and these rates will fall to more acceptable levels (below $10 \%$ at many levels of power) under a 0.005 cutoff.

(ii) Empirical studies of replication rates in psychology [Open Science Collaboration, 2015] and experimental economics [Camerer, et al, 2016] suggest that the replication rate for findings with $P$-value in the range $P<0.005$ is twice that of $P$-values in the complementary range $0.005<P<0.05$.

But in light of the analysis in Sections 3-4 neither claim provides valid support for the RSS conclusion that changing the significance cutoff to $P<0.005$ would "immediately improve" replicability. Claim (i) relies on a theory (Section 2) which tacitly ignores $P$-hacking, and thus does not apply in the context for which the RSS proposal was designed. So while it may be true that FPR greater than $33 \%$ is unacceptably high, the projected decrease in FPR to the range $5-10 \%$ at the 0.005 level holds only in the absence of $P$-hacking. With $P$-hacking accounted for, Figure 2 shows that FPR will likely remain well above $33 \%$ even after the cutoff is changed to 0.005 , and thus will continue to be "unsettling" according to the RSS authors' own standards. And if the new 0.005 cutoff leads to lower power or more $P$-hacking (as discussed in Section 4), then the false positive rate could increase, as shown in Figures 4 and 5. Claim (ii) ignores the dependence of the persistence rate $\psi_{\alpha}$ on the significance cutoff $\alpha$, as supported by empirical studies [Head, et al, 2015, Mariscampo and Lalande, 2012,Simonsohn, et al, 2014] and common sense - since $P$-hackers currently flout prescribed statistical protocol, knowingly or unknowingly, they should be expected to continue doing so when the prescription changes unless the prescription directly addresses the root cause (adverse incentives) of P-hacking and the replication crisis. With the dependence of $\psi_{\alpha}$ 
on the significance cutoff accounted for, we see that the suggested benefits of lowering the significance threshold are far less certain than presented in [Benjamin, et al, 2017]; see, e.g., Figure 4.

Claimed benefits of the RSS proposal are exaggerated. Figure 4 shows how the false positive rate will change (as a function of $\pi$ ) compared to several reference points. The top dotted line in the left-hand, respectively right-hand, plots indicate the FPR for $h=0.05$, resp. $h=0.15$, at the 0.05 significance level. In both cases, the FPR is much higher than standard theory predicts in the absence of $P$-hacking (as given by the middle dotted line in both plots). The bottom dotted line is the FPR predicted at level 0.005 in the absence of $P$-hacking.

The difference between middle and bottom dotted lines in the plots of Figure 4 shows the projected improvement to FPR based on the analysis in [Benjamin, et al, 2017]. The solid curve shows how FPR varies (at the 0.005 cutoff) with $\pi$. Notice that FPR under the new 0.005 cutoff will remain above $20 \%$ even if persistence is relatively low $(\pi \approx 0.25)$. For high levels of persistence (e.g., $\pi \geq 0.50$ ), the FPR is near or far above what would be expected in the absence of $P$-hacking even at the 0.05 level. Overall, the false positive rate remains much higher than the bottom dotted line (the optimistic RSS projection that assumes no $P$-hacking) unless the persistence rate is negligible. The gap between the solid curve and the bottom dotted line shows the discrepancy between the projected improvement to FPR in [Benjamin, et al, 2017] (i.e., bottom line) and the expected FPR with $P$-hacking accounted for (i.e., solid curve). Figure 4 also illustrates the possible impact of increased $P$-hacking that might result from changing the significance cutoff to 0.005 . The solid red curves in Figure 4 show how adverse $P$-hacking (i.e., $h^{\prime}>0$ ) reduces the impact of the RSS proposal even further, and leaves open the possibility that false positive rate could increase under the 0.005 cutoff. More on this point below.

False positive rate will remain high under RSS. Figure 6 gives a finer grained analysis by plotting FPR for different combinations of power and persistence $\psi_{0.005}(0.005)$. The RSS analysis assumes $h=0$, which at $80 \%$ power leads to a decrease in FPR from 0.38 (under the 0.05 cutoff) to 0.06 (under the 0.005 cutoff). These improvements are substantially 

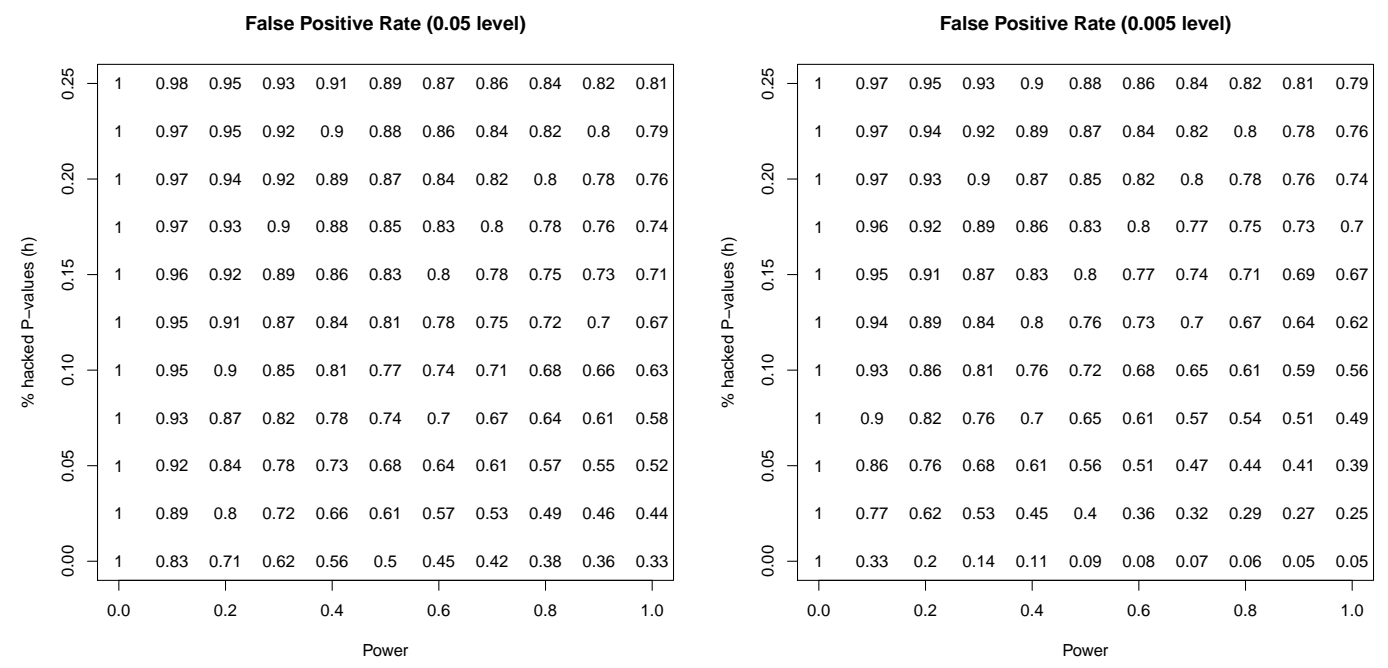

FiguRE 6. False positive rates under different combinations of power $(1-\beta)$ and $P$-hacking rate $(h)$ for significance level 0.05 (left) and 0.005 (right). The prior odds are taken to be $1 / 10$ (i.e., $\phi=10 / 11$ ) in both cases and power at the 0.05 level is fixed at 0.80 .

mitigated in the presence of even modest $P$-hacking: for $h=0.05$ and $80 \%$ power, FPR decreases from 0.57 to 0.44 ; and for $h=0.15$ and $80 \%$ power, FPR decreases from 0.75 to 0.71. Similar relative improvements occur at other levels of power, e.g., at $50 \%$ power, FPR decreases from 0.83 to 0.80 when $h=0.15$ and from 0.68 to 0.56 when $h=0.05$. So if power does not decrease under the lower cutoff, then the false positive rate will go down, but by much more modest factors that are unlikely to be noticeable in practice and would still leave FPR at much higher levels than desired.

The replication crisis could get worse. The possibility that redefining statistical significance could lead to power loss and more P-hacking, as noted in Section 4 and [Amrhein and 490 Greenland, 2017, Lakens, et al, 2017, McShane, et al, 2017], leads to even greater uncertainty about the eventual impact of the proposal to redefine statistical significance. Figures $4-5$ demonstrate a range of possible outcomes depending on whether or not $P$-hacking increases and/or power decreases in response to the lower significance level. The right-hand plot in Figure 5 shows that if $P$-hacking attempts double from $h=0.05$ at current level 0.05 to 0.10 at the 0.005 level, then the replication rate will decrease under the RSS proposal unless power remains close to its current level and $P$-hacking persistence is negligible. Even in 
a best-case scenario, in which persistence is 0 and power remains at $80 \%$, the lower cutoff improves replication rate by only $25 \%$ (the bottom, right-most number in right-hand plot of Figure 5). In many more realistic scenarios (e.g., persistence 50\% and power decreases from $80 \%$ to $60 \%$ ), the replication rate will decrease by about $12 \%$.

\section{Concluding Remarks}

6.1. Summary of conclusions. The RSS proposal has received a great deal of (mostly negative) attention in the time since it first appeared, with a number of authors, e.g., [Amrhein and Greenland, 2017, Hamlin, 2017, Lakens, et al, 2017, Mayo, 2017, McShane, et al, 2017, Perezgonzalez and Frias-Navarro, 2017, Trafimow, et al, 2017], raising the possibility that lowering the significance cutoff would have neutral or negative consequences for the replication crisis. The analyses presented in Sections 3-4 complement many of these prior criticisms by articulating precise scenarios under which their concerns could come to light. Even the authors of [Benjamin, et al, 2017] acknowledge that $P$-hacking and related reporting issues are "arguably the bigger problems" underlying the replication crisis, claiming instead that, while the 0.005 proposal does not directly combat $P$-hacking, "[r]educing the $P$-value threshold complements [...] solutions to these other problems" [Benjamin, et al, 2017]. But the RSS authors fail to appreciate that these other problems, though not treated directly by their proposal, still bear on its eventual impact on replication.

The above analysis casts doubt on the complementary nature of the RSS proposal: there are several plausible scenarios under which the lower threshold could undermine efforts to improve replication; and even if the lower cutoff leads to minor improvements, the added cost may have adverse effects on other more direct efforts. So while the proposal itself may not be intended to combat $P$-hacking, a realistic evaluation of the proposal must account for the existence, and inevitable side effects, of $P$-hacking on the relevant metrics (e.g., false positive rate). I have argued above that by ignoring $P$-hacking and other relevant reporting issues, the RSS authors present a misleading argument and reach misguided conclusions about the impact of their proposal. 
At minimum, my analysis suggests that the consequences of redefining statistical signifito tackle this important issue.

6.2. Is statistics serving its purpose? The past century has seen the steady rise of statistical thinking as a method for validating scientific findings [Porter, 1988]. In many fields, statistics is now the sole adjudicator of what is 'significant' and what is not, what largely been phased out, slowly but steadily [Gigerenzer, et al, 1989, Porter, 1996], to such an extent that it is now difficult or impossible to describe what constitutes 'knowledge' 
in many fields without referring to the concept of statistical significance, or some similar statistical measure. Beyond their implications for the specific recommendation to redefine statistical significance, the subtle but severe issues with the RSS proposal call into question the role of statistics in scientific research, and whether or not the widespread use of statistics in science is serving its intended purpose [Crane and Martin, 2017].

The trouble with ever more reliance on statistical reasoning is evident by comparing my analysis above with that in [Benjamin, et al, 2017]. Granting the authors of [Benjamin, et al, 2017] the benefit of the doubt, we can assume both analyses were conducted honestly and in good faith. And yet the two analyses tell very different stories about the potential impact of the 0.005 proposal on the replication crisis. Evidently, for all but the most straightforward problems, whose relevance is mostly confined to classroom demonstrations, homework assignments, and thought experiments, statistical analysis affords ample degrees of freedom which have nothing to do with the so-called "researcher degrees of freedom" that contribute to $P$-hacking and related scientific reporting issues. Perhaps the bigger problem facing science is not $P$-hacking, misconduct, publication bias, or questionable research practices but rather its unwavering trust in non-scientific implementations of methods from statistics and so-called "data science".

[Amrhein and Greenland, 2017] V. Amrhein and A. Greenland. (2017). Remove, rather than redefine, statistical significance. Nature Human Behaviour.

[Bakker, et al, 2012] M. Bakker, A. van Dijk and J.M. Wicherts. (2012). The Rules of the Game Called Psychological Science. Perspectives on Psychological Science, 7(6), 543-554.

[Benjamin, et al, 2017] D.J. Benjamin, J.O. Berger, Magnus Johannesson, B. Nosek, E.J. Wagenmakers, R. Berk, K.A. Bollen, B. Brembs, L. Brown, C. Camerer, D. Cesarini, C. Chambers, M. Clyde, T. Cook, P. de Boeck, Z. Dienes, A. Dreber, K. Easwaran, C. Efferson, E. Fehr, F. Fidler, A.P. Field, M. Forster, E. George, R. Gonzales, S. Goodman, E. Green, D. Green, A. Greenwald, J. Hadfield, L. Hedges, L. Held, T.H. Ho, H. Hoijtink, J.H. Jones, D. Hruschka, K. Imai, G. Imbens, J. Ioannidis, M. Jeon, M. Kirchler, D. Laibson, J. List, R. Little, S. Lupia, E. Machery, S. Maxwell, M. McCarthy, D. Moore, S. Morgan, M. Munaf, S. Nakagawa, B. Nyhan, T. Parker, L. Pericchi, M. Perugini, J. Rouder, J. Rousseau, V. Savalei, F. Schoenbrodt, T. Sellke, R. Shiffrin, B. Sinclair, D. Tingley, T. Van Zandt, 
S. Vazire, D. Watts, C. Winship, R. Wolpert, Y. Xie, C. Young, J. Zinman, V.E. Johnson. (2017). Redefine statistical significance. Nature Human Behaviour.

[Head, et al, 2015] M.L. Head, L. Holman, R. Lanfear, A.T. Kahn, M.D. Jennions. (2015). The Extent and Consequences of P-hacking in Science. PLOS Biology.

[Ioannidis, 2005] J.P.A. Ioannidis. (2005). Why Most Published Research Findings are False. PLoS Medicine,

[Bishop and Thompson, 2016] D.V.M. Bishop and P.A. Thompson. (2016). Problems in using p-curve analysis and text-mining to detect rate of p-hacking and evidential value. PeerJ.

[Button, et al, 2013] K.S. Button, J.P.A. Ioannidis, C. Mokrysz, B.A. Nosek, J. Flint, E.S.J. Robinson, et al. (2013). Power failure: why small sample size undermines the reliability of neuroscience. Nature Reviews Neuroscience, 14, 1-12.

Johannesson, Michael Kirchler, Johan Almenberg, Adam Altmejd, Taizan Chan, Emma Heikensten, Felix Holzmeister, Taisuke Imai, Siri Isaksson, Gideon Nave, Thomas Pfeiffer, Michael Razen, Hang Wu. (2016). Evaluating replicability of laboratory experiments in economics. Science.

[Chambers, 2014] C. Chambers. (2014). Registered Reports: A step change in scientific publishing. Accessed at https://www.elsevier.com/reviewers-update/story/innovation-in-publishing/registeredreports-a-step-change-in-scientific-publishing on March 22, 2018.

[Crane and Martin, 2017] H. Crane and R. Martin. (2017). Is statistics meeting the needs of science? Accessed at https://psyarxiv.com/q2s5m/ on March 22, 2018.

[Gelman and Loken, 2014] A. Gelman and E. Loken. (2014). Data-dependent analysis - a "garden of forking paths"- explains why many statistically significant comparisons don't hold up. Am. Sci., 102(6):460.

[Fraser, et al, 2018] H. Fraser, T. Parker, S. Nakagawa, A. Barnett, and F. Fidler. (2018). Questionable Research Practices in Ecology and Evolution. Accessed at https://osf.io/ajyqg/ on March 21, 2018.

[Gigerenzer, et al, 1989] G. Gigerenzer, Z. Switjtink, T. Porter, L. Daston, J. Beatty, and L. Krüger. (1989). The Empire of Chance: How probability changed science and everyday life.

[Hamlin, 2017] K. Hamlin. (2017). Comment at http://philosophyofbrains.com/2017/10/02/should-weredefine-statistical-significance-a-brains-blog-roundtable.aspx.

[Hartgerink, 2017] C.H.J. Hartgerink. (2017). Reanalyzing Head et al. (2015): investigating the robustness of widespread p-hacking. PeerJ.

[Hartgerink, et al, 2016] C.H.J. Hartgerink?, R.C.M. van Aert, M.B. Nuijten, J.M. Wicherts and M.A.L.M. van Assen. (2016). Distributions of p-values smaller than .05 in psychology: what is going on?. PeerJ.

$2(8), \mathrm{e} 124$. 
[Ioannidis, 2018] J.P.A. Ioannidis. (2018). The Proposal to Lower P Value Thresholds to .005. Journal of the American Medical Association. Published online March 22, 2018.

[John, et al, 2012] L.K. John, G. Loewenstein, and D. Prelec. Measuring the prevalence of questionable research practices with incentives for truth telling. Psychological Science, 1;23(5):524-32, 2012.

[Perezgonzalez and Frias-Navarro, 2017] J.D. Perezgonzalez and D. Frias-Navarro. (2017). Retract 0.005 and propose using JASP, instead. psyarxiv.com/t2fn8.

[Open Science Collaboration, 2015] Open Science Collaboration. (2015). Estimating the reproducibility of 645 Stat. Assoc., 112, 1-10. Ten simple rules for effective statistical practice. PLOS Comput. Biol., 12(6):e1004961.

[Lakens, et al, 2017] D. Lakens, et al. Justify Your Alpha: A Response to "Redefine Statistical Significance". PsyArXiv, 18 Sept. 2017. Web.

[Mackey, 2012] A. Mackey. (2012). Why (or why not), when, and how to replicate research. In G. Porte (Ed.), Replication research in applied linguistics (pp. 21-46). New York: Cambridge University Press.

[Mariscampo and Lalande, 2012] E.J. Mariscampo and D.R. Lalande. (2012) A peculiar prevalence of p values just below .05. Q Rev Biol. 65:2271-2279.

[Maxwell, 2004] S.E. Maxwell. (2004). The persistence of underpowered studies in psychological research:

[Maxwell, et al, 2015] S.E. Maxwell, M.Y. Lau, and G.S. Howard. (2015). Is psychology suffering from a replication crisis? What does "failure to replicate" really mean? American Psychologist, 70, 487-498.

[Mayo, 2017] D. Mayo. (2017). Comment at http://philosophyofbrains.com/2017/10/02/should-we-redefinestatistical-significance-a-brains-blog-roundtable.aspx.

[McShane, et al, 2017] B.B. McShane, D. Gal, A. Gelman, C. Robert, J.L. Tackett. (2017). Abandon statistical significance. arXiv:1709.07588.

[Neyman and Pearson, 1933] J. Neyman and E.S. Pearson. (1933). On the Problem of the Most Efficient Tests of Statistical Hypotheses. Philosophical Transactions of the Royal Society A, 231:289-337.

[Nuitjen, et al, 2016] M.B. Nuijten, C.H.J. Hartgerink, M.A.L.M. van Assen, S. Epskamp, and J.M. Wicherts, J. M. (2016). The prevalence of statistical reporting errors in psychology (1985-2013). Behavior Research Methods, 48, 1205-1226. psychological science. Science, 349:6251.

[Porter, 1988] T.M. Porter. (1988). The Rise of Statistical Thinking: 1820-1900.

[Porter, 1996] T.M. Porter. (1996). Trust in Numbers. 
[Simmons, et al, 2011] J.P. Simmons, L.D. Nelson and U. Simonsohn. (2011). False-Positive Psychology Undisclosed Flexibility in Data Collection and Analysis Allows Presenting Anything as Significant.

650

[Simonsohn, et al, 2014] U. Simonsohn, L.D. Nelson, and J.P. Simmons. (2014) P-Curve: A Key to the File Drawer. Journal of Experimental Psychology.

[Szucs and Ioannidis, 2017] D. Szucs and J.P. Ioannidis. (2017). Empirical assessment of published effect sizes and power in the recent cognitive neuroscience and psychology literature. PLoS Biology, 15, e2000797.

[Trafimow, et al, 2017] David Trafimow, Valentin Amrhein, Corson N. Areshenkoff, Carlos Barrera-Causil, Eric J. Beh, Yusuf Bilgiç, Roser Bono, Michael T. Bradley, William M. Briggs, Héctor A. CepedaFreyre, Sergio E. Chaigneau, Daniel R. Ciocca, Juan Carlos Correa, Denis Cousineau, Michiel R. de Boer, Subhra Sankar Dhar, Igor Dolgov, Juana Gómez-Benito, Marian Grendar, James Grice, Martin E. Guerrero-Gimenez, Andrés Gutiérrez, Tania B. Huedo-Medina, Klaus Jaffe, Armina Janyan, Ali Karimnezhad, Fränzi Korner-Nievergelt, Koji Kosugi, Martin Lachmair, Rubén Ledesma, Roberto Limongi, Marco Tullio Liuzza, Rosaria Lombardo, Michael Marks, Gunther Meinlschmidt, Ladislas Nalborczyk, Hung T. Nguyen, Raydonal Ospina, Jose D. Perezgonzalez, Roland Pfister, Juan José Rahona, David A. Rodríguez-Medina, Xavier Romão, Susana Ruiz-Fernández, Isabel Suarez, Marion Tegethoff, Mauricio Tejo, Rens van de Schoot, Ivan Vankov, Santiago Velasco-Forero, Tonghui Wang, Yuki Yamada45, Felipe C. Zoppino, Fernando Marmolejo-Ramos. (2017). Manipulating the alpha level cannot cure significance testing - comments on "Redefine statistical significance". PeerJ Preprints.

[Trafimow and Marks, 2015] D. Trafimow and M. Marks. (2015). Editorial. Basic and Applied Social Psychology, 37:1-2.

[Wicherts, et al, 2016] J.M. Wicherts, C.L. Veldkamp, H.E. Augusteijn, M. Bakker, R.C. van Aert, and M.A. van Assen. (2016). Degrees of Freedom in Planning, Running, Analyzing, and Reporting Psychological Studies: A Checklist to Avoid p-Hacking. Front Psychol, 7, 1832.

[Ziliak and McCloskey, 2008] S.T. Ziliak and D.N. McCloskey. (2008). The Cult of Statistical Significance: How the Standard Error Costs Us Jobs, Justice, and Lives (Economics, Cognition, and Society). 